\title{
Last 2 Days
}

National Cancer Institute

\section{Source}

National Cancer Institute. Last 2 Days. NCI Thesaurus. Code C136324.

The 48 hour period occurring just before the present time. 